\title{
La investigación sobre violencia contra las mujeres en América Latina: entre el empirismo ciego y la teoría sin datos
}

\author{
Research on violence against women \\ in Latin America: from blind empiricism \\ to theory without data
}

Roberto Castro 1

Florinda Riquer 2

\footnotetext{
1 Centro Regional de Investigaciones Multidisciplinarias, Universidad Nacional Autónoma de México. Apartado Postal 4-106, C. P. 62431, Cuernavaca, Morelos, México. rcastro@servidor.unam.mx 2 Universidad Iberoamericana Prolongación Paseo de la Reforma 880, C. P. 01210 , México, DF, México.
}

\begin{abstract}
Research on violence against women in Latin America presents an interesting paradox: while the number of studies is quite small, there also appears to be a sense that research on this topic has been exhausted, despite the lack of any definitive responses to the nature and causes of the problem. This results from the boom in studies with a strong empirical focus, lacking any basis in more general sociological theory. On the other hand, research using social theory tends to ignore the existing mediations between structural arrangements and any individual specific behavior, as well as the interactive nature of domestic violence. Meanwhile, empirical research presents inconsistent results and tends to run into methodological problems such as operational confusion, contradictory findings, and results and recommendations that are too obvious. New research designs must be developed to enrich the field and which are solidly based on the body of conceptual knowledge in social sciences, abandoning designs without theory and those which are merely statistical. Only then will it be possible to imagine the new research questions that the problem of violence requires.
\end{abstract}

Key words Domestic Violence; Women's Health; Methods

Resumen La investigación en torno a la violencia contra las mujeres en América Latina enfrenta una paradoja: el número de investigaciones es sumamente bajo, al mismo tiempo que existe una suerte de agotamiento del tema sin haberse dado aún respuestas definitivas sobre la naturaleza y las causas del problema. Ello responde a la orientación predominantemente empírica de las investigaciones, y a su falta de anclaje en la teoría sociológica más general. La investigación que se apoya en la teoría social suele ignorar las mediaciones existentes entre los arreglos estructurales y la conducta específica de los individuos, así como el carácter interaccional de la violencia. La investigación empírica, por su parte, presenta resultados inconsistentes y suele incurrir en problemas metodológicos tales como confusión operacional, y obviedad de los hallazgos y de las recomendaciones. Para enriquecer la investigación es indispensable diseñar nuevas investigaciones sustentadas en el corpus conceptual de las ciencias sociales y abandonar los diseños ateóricos y meramente estadísticos. Sólo así será posible imaginar las nuevas preguntas de investigación que requiere el problema de la violencia.

Palabras clave Violencia Doméstica; Salud de las Mujeres; Métodos 


\section{Introducción}

Este texto surge de la necesidad de hacer un alto en el camino. Tras varios años de participar en la discusión e investigación sobre violencia de género, así como de insistir en su importancia como tema de salud pública (Castro et al., 2000; Castro \& Ruiz, 2002; Riquer, 1992, 1995a, 1995b, comunicación personal; Riquer et al., 1996), nos pareció importante hacer un balance. El objetivo central de este trabajo es mostrar las principales dificultades, teóricas y metodológicas, por las que atraviesa la investigación sobre violencia contra las mujeres en América Latina realizada en las dos últimas décadas.

Cabe advertir que nuestro ejercicio no constituye un Estado del Arte toda vez que no fue posible acceder a la totalidad de las investigaciones sobre el tema, en gran medida porque sus resultados no se publican. Por ello, los trabajos seleccionados (más de 70) para realizar este ejercicio de análisis representan, en todo caso, líneas de preocupación y modos de investigar el complejo problema de la violencia contra las mujeres en nuestro continente.

Con fines analíticos proponemos que es posible diferenciar tres grandes vías de aproximación al problema de la violencia contra las mujeres: los acercamientos teóricos, la investigación empírica y las intervenciones para la atención de las mujeres. Nuestra impresión es que tales aproximaciones se han desarrollado de manera independiente entre sí, esto es, con muy pocos - o nulos - puntos de contacto. Sostenemos que la investigación sobre el problema de la violencia contra las mujeres participa del trágico atributo que Bourdieu señala como distintivo de la investigación social en muchos países: "quizás nunca ha sido tan grande como hoy en día la distancia entre lo que en los Estados Unidos y en todos los países dominados por el modelo académico americano se llama teoría y lo que se denomina investigación empírica" (Bourdieu, 2000a:64-65). Este es el argumento que queremos desarrollar en este trabajo. Para ello, en la primera parte del texto, reflexionamos sobre las posibles causas de tal disociación y se sintetizan, muy someramente, los principales intentos de conceptualización. En la segunda parte, se presentan los principales resultados de los llamados estudios empíricos. En la tercera parte se señalan algunos de los principales problemas metodológicos de tales estudios. Al final, apuntamos algunos caminos para enriquecer la mirada teórica sobre el fenómeno y mejorar las aproximaciones empíricas.
De la urgencia de la intervención

a la conceptualización: ¿un salto al vacío?

La violencia contra las mujeres (en este trabajo sinónimo también de "violencia de género") es uno de los ejes de reflexión - acción con lo que resurge el feminismo en América Latina a finales de los años sesenta del siglo XX (Anonymous, 1984-1985, 1987, 1992; Barroso, 1988; Bedregal, 1991a). El feminismo contribuyó de manera decisiva a visualizar el fenómeno como un problema social (no sólo individual), de orden público (no únicamente privado), de competencia de los tribunales, y que exige políticas públicas con obligaciones específicas para las instituciones, incluidas las de salud.

En nuestra perspectiva, en gran medida puede comprenderse el desarrollo del tema como objeto de conocimiento y la disociación entre intentos de conceptualización, investigación empírica y propuestas de atención, en tanto no surge de alguna de las ciencias sociales, ni del ámbito de las ciencias de la salud. Surge, como todos los temas que fueron dando cuerpo al campo de conocimiento de los estudios sobre la mujer, de la urgencia sentida por algunas mujeres de atender a las víctimas de la violencia de género, por evidenciar la importancia social y política de la problemática y de pugnar por generar marcos jurídicos y políticas públicas adecuadas para hacerle frente al problema. Esto significa que los temas derivados de dicha agenda fueron entrando al mundo académico en medio de un amplio recelo y con dificultades para legitimarse.

Así, principalmente en México, Brasil y Perú, algunas mujeres tomaron por su cuenta la atención de otras, víctimas, en concreto, de violación. Crearon centros que brindaban atención psicológica, generalmente combinada con asesoría legal. Estamos hablando de un momento en el que la legislación sobre violación en nuestros países y los mecanismos institucionales para su denuncia eran incipientes e inadecuados. Por ello, en paralelo, algunas mujeres se dieron a la tarea de revisar las disposiciones legales y hacer las primeras propuestas de reformas (Bedregal, 1991b; Mogrovejo, 1989).

La propia práctica condujo a algunos grupos de activistas a realizar algún nivel de sistematización de sus "casos" (Bedregal et al., 1991; Duarte, 1992; Rojas, 1991; Saucedo, 1991; Silva, 1986), así como a realizar los primeros estudios sobre la magnitud y prevalencia de la violencia contra la mujer (Bedolla, 1987; Granados, 1996; Larráin \& Rodríguez, 1993; PROFAMILIA, 1990; Ramírez \& Uribe, 1993; Valdez \& Shrader, 1992). Tanto la sistematización como los estudios pa- 
recen haber estado más inspirados en contar con elementos para la denuncia pública y política del fenómeno, que por un afán de conocer más y mejor la problemática.

En suma, durante más de dos décadas, feministas de varios países de la región latinoamericana intentaron hacer "visible" la violación y el hostigamiento sexual; más tarde, en la década de los noventa del siglo pasado, se ocuparon de visibilizar la violencia doméstica o intrafamiliar y una serie de prácticas ancestrales como la mutilación femenina, el infanticidio de niñas y el feminicidio. Hoy sabemos que el esfuerzo no ha sido en vano.

Por una parte, se cuenta ya con una serie de documentos internacionales que obligan a los países que los han firmado a implementar medidas para hacer frente a la violencia de género. Por lo menos entre los organismos que componen el sistema de Naciones Unidas, se ha logrado consenso en torno a la importancia del problema y a la necesidad de hacerle frente. Por otra parte, se cuenta ya con algunos estudios para países de América Latina que señalan la magnitud de la problemática y de sus consecuencias para el desarrollo económico, el bienestar familiar y la salud de las mujeres de todas las edades. Contamos, de igual modo, con algunas aproximaciones cualitativas al fenómeno que van permitiendo avanzar en la descripción de la problemática (Burín \& Meler, 1998; Oliveira, 1998; Saucedo, 1996; Szasz, 1999).

Ahora bien, tras el esfuerzo por visibilizar la violencia contra la mujer como problema social ¿qué conceptualizaciones o comprensiones del fenómenos se han sustentado?. Pocos consensos ha habido al interior del debate feminista, que no es un movimiento con una sola visión o perspectiva, sobre el por qué de la subordinación femenina. Uno de ellos ha sido acerca de la causa última de la violencia contra la mujer. Esa causa se denominó "patriarcado"; la violencia contra las mujeres ha sido concebida, entonces, como la expresión más brutal del fenómeno llamado patriarcado.

Si bien el término patriarcado no ha estado exento de controversia (Castro \& Bronfman, 1993; Rubin, 1975), el desarrollo de la teoría feminista y el nuevo conocimiento generado en el campo de los estudios de género autorizan a suponer que el patriarcado es un fenómeno de carácter estructural, constitutivo y constituyente del orden y la reproducción de las sociedades. Concebido así, en toda sociedad están dadas las condiciones para que cualquier mujer viva en riesgo de ser violada, toda esposa de ser golpeada, toda empleada y estudiante hostigada sexualmente por un superior. Al tiempo que todo varón está en riesgo de constituirse o convertirse en un violador, un golpeador o un hostigador.

En clave política, afirmar que el patriarcado es la causa última de la violencia contra la mujer ha tenido alguna eficacia, sobre todo en la medida en que alertó a organismos de Naciones Unidas sobre la gravedad del problema. Sin embargo, como explicación del fenómeno es, a todas luces, insuficiente. Una causa última, aún pudiendo mostrarse que lo es, no necesariamente constituye la explicación del fenómeno en cuestión.

A la luz del material revisado, encontramos una paradoja. A pesar de haber cierto consenso acerca de que la violencia de género es consecuencia del patriarcado, a la hora de estudiar empíricamente la violencia en la pareja y la intrafamiliar, el patriarcado acabó siendo reducido a variables individuales, esto es, reducido a las características sociodemográficas (edad, estado conyugal, escolaridad, ocupación, ingreso) y a aspectos conductuales (principalmente consumo de alcohol y de drogas) del varón. Tal paradoja constituye una falacia metodológica, que consiste, justamente, en reducir un fenómeno de carácter estructural - el patriarcado a indicadores individuales. Esto es, en el estudio de la violencia de género no se ha considerado, o no se sabe que hay que considerar, las mediaciones entre fenómenos a los que se les atribuye carácter estructural, como el del patriarcado y su expresión en la conducta individual. Dicha reducción, a su vez, contribuyó a crear una suerte de caricatura de la violencia de género. En tal caricatura aparecen dos personajes: de un lado, un hombre, casi siempre de baja escolaridad, de escasos recursos económicos y/o desempleado, que a la menor provocación, o sin ella, arremete psicológica y con frecuencia físicamente contra una mujer. Del otro lado, estaría la mujer como víctima, receptora casi pasiva, de la violencia.

Uno de los problemas que supone esta visión caricaturizada es que ella no da cuenta de la condición o situación en la que ocurre, específicamente, la violencia en la pareja. De ahí, quizá que se avanzara en colocar en el centro de la conceptualización ya no a los individuos que participan en la trama de la violencia, sino a la familia (Fassler, 1999). Este desplazamiento ha sido importante en tanto la familia es una de las mediaciones más relevantes entre la conducta individual y los fenómenos de carácter estructural. El desplazamiento permitió, por tanto, observar que, si bien el fenómeno llamado patriarcado constituye una condición general de riesgo de que cualquier mujer sea trata- 
da con violencia y de que cualquier hombre sea un agresor, se requieren de determinados vínculos e interacciones para que los individuos hombres y mujeres - entren en la trama de la violencia de género. Haber puesto en el centro de observación a la familia permitió avanzar desde concebir a la violencia contra la mujer como un problema de conducta individual del varón, a pensarla como resultado de un desequilibrio de poder entre individuos (Corsi, 1994; Giffin, 1994; Heise, 1994) o como resultado de la tensión entre el poder del hombre y la resistencia de la mujer (The British Council, 1999).

Concebir al fenómeno de esa manera no necesariamente ha significado que, en la investigación empírica, ni en la intervención, se establezcan las variables que darían cuenta del desigual acceso y uso a diversos recursos y, por ende, al poder, por parte de cada género (Alves-Cabral, 1999). Como se tratará de documentar en el siguiente apartado, las variables que se siguen considerando son sociodemográficas, económicas y relacionadas con la conducta individual de los varones. Sostenemos que una vía para lograr un acercamiento entre los desarrollos teóricos y las investigaciones e intervenciones concretas pasa por superar las visiones atomistas de lo social que, al parecer, subyacen a la investigación empírica. Para ello, consideramos, hace falta pensar sociológicamente la violencia contra la mujer.

Un primer paso para ello es considerar que los hombres y las mujeres concretos son a la vez productores y portadores de sociedad (Giddens, 1995). Por tanto, nuestra cognición, nuestra conducta y nuestras interacciones son expresión de las cogniciones y de las prácticas dominantes en un tiempo y espacio determinado. En segundo término, y al margen de la discusión sobre si hay o no un componente "naturalmente" violento o agresivo en la constitución de la identidad masculina (Ramírez, 2000; Riquer, 1991), sociológicamente, la dimensión del fenómeno que interesa destacar es la relacional. Si bien consideramos a la violencia y a la agresión como actos o conductas del individuo, suponemos, también, que son medios o mecanismos de expresión de percepciones, necesidades, deseos, afectos y sentimientos que quizá no pueden ser verbalizados y actuados por vías que no dañen a otro e incluso al propio agresor. Son medios o mecanismos que suponen la presencia de otro, y, en ese sentido, consideramos a la agresión y a la violencia más como un aspecto o forma que la relación entre dos o más sujetos asume que como una característica del individuo. Por ello, es necesario plantearse preguntas distintas a las que pare- cen haber guiado la investigación empírica. Básicamente lo que se ha preguntado es cuántas mujeres son violadas, agredidas física, sexual o emocionalmente por sus parejas, $u$ hostigadas en la escuela o la oficina; y cuáles son las características de los agresores. Pero, si se acepta que la violencia de género es un problema relacional y específicamente de acceso y uso desigual de diversos recursos entre los integrantes de una pareja y/o al interior del hogar, sobre lo que habría que preguntar es acerca del poder en las relaciones de género. Asunto hace tiempo planteado en la teorización feminista que sigue en busca de un nicho dentro de la teoría sociológica y que aún no se traduce en variables e indicadores que permitan mejorar nuestra comprensión de la violencia contra las mujeres.

\section{La investigación empírica}

Como se ha señalado ya, ésta no pretende ser una revisión exhaustiva de la literatura empírica sobre el problema de la violencia contra las mujeres en América Latina. En esta sección, nuestro análisis se centrará básicamente en los estudios que buscan explorar las causas de la violencia contra las mujeres, esto es, qué factores la producen, y dejaremos de lado los estudios sobre las consecuencias de la misma.

\section{El problema de la prevalencia de la violencia contra las mujeres}

Lo que primero ha preocupado a los investigadores latinoamericanos se refiere a la magnitud del problema de la violencia. Las prevalencias reportadas presentan cierta variación pero normalmente dentro de un determinado rango. Algunas de las primeras investigaciones de la década pasada reportaban prevalencias superiores al 50\% (60\% en Chile, según Larráin, 1993; 54\% en Costa Rica, según Chacón et al., 1990, apud Heise, 1994; 57\% en el área rural de Jalisco, México, según Ramírez \& Uribe, 1993; $56 \%$ en Guanajuato, México, según Tolbert \& Romero, 1996). Al paso de los años, quizás como efecto de un mejor diseño de los instrumentos de medición, las prevalencias reportadas se han centrado en un rango que va del $10 \%$ al 52\% (26\% en Colombia, según Klevens, 2001; 46\% en Guadalajara, México, según Ramírez \& Patiño, 1996; 42\% en Durango, México, según Alvaro-Zaldívar et al., 1998; y 52\% en Nicaragua, según Ellsberg et al., 1999, 2000).

Cabe, sin embargo, hacer una crítica general a los estudios de prevalencia. La mayoría de ellos no reportan haber diferenciado a la vio- 
lencia que sufren las mujeres entrevistadas en términos de severidad. Ello hace que los autores reporten como "prevalencia" lo mismo a casos de mujeres que sufren formas "moderadas" de violencia episódica, que a casos de mujeres que, en el otro extremo, reportan sufrir formas graves de violencia crónica. Ello significa que el rango de prevalencias reportado en la actualidad (que oscila, como dijimos, entre un $10 \%$ y $50 \%$ ) constituye en muchos casos un dato poco preciso. Pues una mujer que reporta haber sufrido una vez una bofetada de parte de su pareja - y no queremos minimizar la gravedad de cualquier forma de violencia contra las mujeres - se puede parecer más, para fines analíticos, a las mujeres que nunca han recibido una agresión física, que a aquellas que la han experimentado de manera grave y variada durante años. A menos que se introduzcan índices de severidad que permitan distinguir tanto a las mujeres que sufren violencia moderada de las que sufren violencia severa, como a las que sufren violencia episódica de las que sufren violencia crónica, seguiremos obligados a considerar a las tasas de prevalencia como indicadores más bien débiles, con valor sólo indicativo, de la gravedad del problema de la violencia contra las mujeres.

\section{Variables más comúnmente} asociadas a la violencia

Al tomar al problema de la prevalencia como una cuestión medular, pocos estudios publicados han optado por un diseño más sociológico para la "explicación” del problema. A ello se debe el relativo desorden que se advierte en el listado de variables que se reportan como asociadas de manera estadísticamente significativa al problema de la violencia. Las variables más frecuentemente citadas en esta calidad son el nivel socioeconómico, la escolaridad y el grupo de edad (en todos los casos mientras más bajo, más riesgo), el estado civil (las mujeres no casadas en más riesgo), el haber sufrido abuso o violencia durante la infancia o el haber sido testigo de ella (tanto la mujer como su pareja), la condición de ocupación de la pareja (si está desempleado más riesgo) así como de la mujer (si trabaja fuera de la casa menos riesgo); el número de hijos, el número de años de la unión, y el consumo de alcohol (en los tres casos, mientras más alto más riesgo de violencia); y finalmente la existencia de una marcada asimetría de poder en la pareja, así como la existencia de una ideología de los roles de género (Buvinic et al. 1999; Deslandes, 2000; Díaz-Olavarrieta et al., 1999, 2001; J. Firestone, R. Harris \& W. Vega, comunicación personal; Garza \& Díaz-Michel, 1997; Meneghel et al., 2000; Moreno, 1999).

En la mayor parte de los casos, las estrategias preferidas son el análisis estadístico bivariado y los modelos de regresión logística. Más allá de que siempre es útil identificar las variables que se asocian estadísticamente a la violencia que sufren las mujeres, no es difícil advertir el limitado alcance de estos trabajos. Al carecer de una teoría sociológica que ordene y jerarquice a las variables relevantes a partir de las categorías de género y poder - en el marco de una teoría que articule al actor con la estructura y la historia - estos trabajos sólo aciertan a reportar la fuerza de las asociaciones encontradas. Pero más grave aún, al carecer de un marco así, estos estudios no suelen aclarar los criterios que llevaron a los autores a explorar ciertas variables y no otras en su asociación con la violencia.

Quizás a ello se debe la doble preocupación que inevitablemente se tiene al revisar esta literatura: por una parte, la sensación de que no se está acumulando conocimiento nuevo en esta línea desde hace ya varios años, pues los diversos estudios tienden a reportar cosas semejantes; y por otra parte, la sensación de que se ha procedido con cierta arbitrariedad en la selección de las variables a estudiar (seguramente haciendo eco a estudios previos), lo que a su vez está limitando el desarrollo de otros diseños que de manera bien fundamentada se arriesguen a explorar en nuevas direcciones.

Otros estudios, más centrados en mujeres de poblaciones específicas, permiten establecer comparaciones, en términos de prevalencia, entre mujeres de diversos grupos, o entre mujeres de un grupo en particular con respecto a la población en general. Este tipo de comparaciones permite conocer si determinado grupo de mujeres está expuesto a un riesgo mayor de violencia que el resto. Sin que siempre sea éste el objetivo explícito de los autores, este tipo de trabajos permite una lectura más sociológica de los resultados, debido a que trabajan con grupos sociales, más que con mujeres individuales agrupadas artificiosamente mediante meros procedimientos estadísticos. A esta corriente pertenecen, entre otros: (a) los estudios que exploran la prevalencia de violencia entre mujeres embarazadas, (b) los estudios que se centran en mujeres con problemas específicos de salud, (c) los estudios con mujeres atendidas por los servicios públicos de salud, y (d) los estudios con mujeres de diferentes grupos étnicos, etc.

Respecto a las mujeres embarazadas, existen en la región varios estudios que buscan ex- 
plorar si el embarazo constituye un factor de riesgo o, a la inversa, un factor de protección frente a la violencia masculina. Más allá de que se trata de un grupo de población vulnerable al que interesa proteger, la idea de centrarse en las mujeres embarazadas no carece de fundamento sociológico: el embarazo es un rol social bien establecido que genera expectativas específicas en la población (Miller, 1978). La hipótesis subyacente - de la que ciertamente no todos los autores parecen estar al tanto - es que, de encontrarse un patrón en la relación embarazo-violencia, es posible rastrear en los atributos del rol algunas de las explicaciones de este problema, o bien, identificar otros roles sociales que también se asocian a la violencia y explorar si poseen todos ellos algo en común.

Los resultados, sin embargo, como ha sido señalado ya para otros países (Heise, 1994; Moore, 1999), no pueden ser más confusos. Por ejemplo, una investigación reciente en Chile encontró claramente que el embarazo funciona como un factor de protección (Arcos et al., 2000). Simultáneamente, una investigación realizada por nosotros mismos en México (Castro \& Ruiz, 2002) muestra que, en términos de prevalencia, el embarazo no hace ninguna diferencia, toda vez que, tanto antes como durante el embarazo se encontró una prevalencia del $25 \%$. Al mismo tiempo, en otras latitudes se reporta que el embarazo constituye, al contrario, un factor de riesgo para la violencia (Goodwin et al., 2000).

La función del embarazo con relación a la prevalencia de violencia sigue siendo, pues, un problema científico que requiere de mucha más investigación. Esta debe considerar tres cuestiones muy importantes para avanzar. Primero, que las prevalencias reportadas en este ámbito son, en última instancia, similares a las reportadas entre las mujeres no embarazadas; por ejemplo, un estudio entre mujeres embarazadas de Nicaragua (Ellsberg et al., 2000) encontró una prevalencia de $31 \%$, de manera que la medición de las diferencias, si las hay, exige de una precisión que hasta ahora no se ha alcanzado. Segundo, que la diferencia que puede hacer el embarazo no necesariamente debe ubicarse en términos de prevalencia, sino que puede ser incluso más importante la diferencia en términos de severidad. En efecto, en la investigación previamente citada (Castro \& Ruiz, 2002), encontramos que, si bien la prevalencia se mantiene constante antes y durante el embarazo, la severidad de la violencia varía por tipo de violencia: mientras que la severidad de la violencia física y sexual disminuye durante el embarazo, la severidad de la violencia emocio- nal aumenta significativamente. Y tercero, que la indagación sociológica en esta línea debe resistir a la tentación de limitarse a la mera exploración de asociaciones; debe, por el contrario, adentrarse en el ámbito de la teoría social y desde ahí formular las preguntas que guíen la investigación. Por ejemplo: ¿la condición de embarazada traduce un cambio en la identidad - adscrita y autopercibida - de parte de las mujeres? O bien: ¿la condición de embarazo traduce un cambio en el nivel del conjunto de saberes y prácticas de la pareja? Si es así, ¿en qué dirección se dan esos cambios, y cómo se asocian con los patrones de violencia detectados?

Los estudios que se centran en mujeres con problemas específicos de salud pueden ayudar a explorar si esta condición, la de la enfermedad, constituye un factor de riesgo adicional para las mujeres frente a la violencia. Al igual que en el caso anterior, el recorte del objeto de estudio no carece de fundamento, no sólo porque las mujeres enfermas son también un grupo vulnerable con derecho a la protección, sino porque el del enfermo es - bien lo sabemos - un complejo rol social.

Aquí los resultados también son inciertos: al parecer, algunas condiciones de salud, como el SIDA, se asocian con un mayor riesgo de sufrir violencia (Herrera \& Campero, 2000), mientras que otras, como el padecimiento de desórdenes neurológicos crónicos (Díaz-Olavarrieta et al., 1999) parecen asociarse, según los estudios disponibles, a tasas de prevalencia semejantes a las reportadas para la población en general (cerca del 30\%).

Es evidente que esta línea de investigación requiere ser enriquecida. La indagación desde esta perspectiva debe nutrirse de los esclarecedores avances logrados en el campo de la enfermedad como rol social. Desde ahí hemos aprendido que, en tanto que la enfermedad es una entidad sujeta a diversas formas de control social, el concepto de rol del enfermo es "un poderoso instrumento para el análisis de la cultura y de la desviación social" (Turner, 1987:58). En consecuencia, es posible hipotetizar que ciertos padecimientos y ciertas condiciones de salud de las mujeres se asocian a un mayor riesgo de sufrir violencia, mientras que otros padecimientos pueden no generar ningún efecto (ni protector ni de riesgo). Si es así, ¿cómo se agrupan esos padecimientos, en términos de sus propiedades sociológicas? ¿qué hay de común al interior de cada grupo de esos padecimientos que pueda sugerir una explicación sobre el efecto que producen con relación a la violencia?

Los estudios que se centran en la violencia que sufren las mujeres en los servicios públi- 
cos de salud constituyen una oportunidad excepcionalmente valiosa para explorar la lógica que articula los mecanismos tanto estructurales como interaccionales que facilitan la aparición de la violencia. Son pocos, sin embargo, los trabajos de este tipo de los que tenemos noticia. El más relevante hasta la fecha (CLADEM, 1998) documenta los mecanismos de violación de los derechos humanos, sexuales y reproductivos de las mujeres a manos del personal de salud, así como las estrategias de encubrimiento y obstaculización de la justicia que despliegan las instituciones. También se ha documentado la existencia de serias agresiones contra las mujeres en los servicios de salud (Diniz \& d'Oliveira, 1998). Y nosotros mismos estamos llevando a cabo ahora una investigación en las salas de labor y parto de dos hospitales públicos de Cuernavaca (México), con miras a documentar las diversas formas en que se violan los derechos de las mujeres en ese lugar, así como los mecanismos de diverso tipo que lo hacen posible (Castro \& Erviti, 2002).

En las instituciones de salud, se pueden observar con claridad varios mecanismos que se asocian estrechamente a la producción de la violencia: la agrupación y el funcionamiento de los médicos en el marco de una profesión; las estrategias de complicidad que ese marco permite; la difusión de un discurso que legitima una forma de hacer medicina (la occidental) y descalifica a las otras; la existencia de jerarquías rígidas e incuestionables entre médicos y pacientes; la introyección, por parte de las mujeres, del discurso dominante (la forma extrema de la violencia simbólica, según Bourdieu); etc.

La investigación en esta línea tiene dos rutas por recorrer: por una parte, se requiere realizar muchos más estudios, en instituciones de salud de diversos niveles de atención, públicas y privadas, con el fin de explorar y combatir esta forma especifica de violencia contra las mujeres. Pero, por otra parte, debe proveer de conceptos y modelos a la investigación general sobre violencia contra las mujeres. ¿Qué formas de complicidad les permite - a los médicos en el hospital, a los hombres en la sociedad - perpetuar su violencia contra las mujeres? ¿Qué discurso ideológico los legitima o encubre? ¿Cuáles son los mecanismos de internalización del discurso de la violencia masculina por parte de las mujeres? Sostenemos que, a partir de una exploración más sistemática del fenómeno de la violencia contra las mujeres en escenarios acotados como las instituciones de salud, es posible derivar conceptos sensibilizadores o "direcciones en las cuáles mirar" (diría Blumer) para explorar el problema, en términos sociológicos, en la sociedad en general.

Finalmente, los estudios que comparan la prevalencia de la violencia entre mujeres de distintos grupos étnicos contienen un potencial explicativo aún pendiente de agotar. Un diseño común suele explorar el papel de la "aculturación” en relación con la violencia. Si bien estos estudios se han realizado sobre todo en Estados Unidos, los que aquí comentamos han incluido a mujeres provenientes de varios países de América Latina, razón por la cuál resultan de nuestro interés.

Los resultados, sin embargo, también aquí son inciertos. Por ejemplo, una investigación en Arizona entre mujeres embarazadas originarias de México encontró que a mayor grado de aculturación de las mismas mayor era la incidencia de violencia, incluso en comparación con las mujeres que permanecieron en México (Mattson \& Rodríguez, 1999). Otro estudio, en cambio, también con mujeres de origen mexicano pero en el estado de California, encontró que la prevalencia de violencia durante el embarazo es prácticamente igual tanto en California como en Morelos (entre $26 \%$ y $29 \%$ ), mientras que la severidad es mayor en Morelos: es decir, la aculturación, en este caso, sí, está sirviendo de factor protector entre las mujeres mexicanas residentes en California (Castro et al., 2001).

Otros trabajos han analizado las características de las mujeres golpeadas de origen latino en comparación con las de las mujeres de origen anglosajón, en diversas ciudades de Estados Unidos. Se reporta que víctimas de origen latino son significativamente más jóvenes, con menor nivel de escolaridad, y más pobres que las mujeres anglosajonas (Ellsberg et al., 1999). Lo cuál quiere decir que, entre las anglosajonas, la violencia se asocia más débilmente con la edad, la educación y la clase social. ¿Qué explica entonces, entre estas últimas, la existencia de la violencia? La información, con todo y su carácter exploratorio, parece sugerir que la violencia no siempre es función de las variables más establecidas en la literatura; y que, por el contrario, las variables relevantes varían entre un grupo étnico y otro. La comparación entre mujeres de diversa procedencia étnica permite orientar la investigación buscando en el dominio de lo cultural las explicaciones requeridas. Y si bien algunas investigaciones sugieren tímidamente esta posibilidad (Gaffney et al., 1997), otras, en cambio, han adoptado una estrategia netamente antropológica (Perez, 2000), o cualitativa (Watts \& Shrader, 1998), pero al costo de renunciar a la posibilidad de rea- 
lizar comparaciones estadísticas entre dos o más poblaciones diferentes. La investigación en esta línea puede enriquecerse enormemente, pensamos, a partir de una adecuada integración de métodos cuantitativos (que recuperen los datos "duros" en términos de prevalencia, incidencia y fuerza de las asociaciones entre variables) y cualitativos (que exploren en profundidad la lógica de las normas, los valores y las diversas expresiones culturales que se asocian a la producción de la violencia).

En síntesis, es poco lo que se puede concluir en términos de conocimientos sólidos logrados hasta ahora en torno a las causas de la violencia. Además de la falta de contenido sociológico en los reportes sobre el tema, existe otra serie de problemas que caracteriza a mucha de esta investigación, misma que discutiremos brevemente en el siguiente apartado.

\section{Problemas vigentes}

\section{Confusión operacional}

En la búsqueda de variables "independientes" que puedan explicar el problema de la violencia contra las mujeres, algunos autores incurren en lo que se denomina "confusión operacional”. Esto no significa más que confundir, al momento de operacionalizar, a las variables independientes con la variable dependiente. Por ejemplo, una investigación reporta que "la frecuencia de conflictos entre la pareja” y la existencia de un alto número de "prohibiciones" de parte de los hombres hacia sus parejas, son las que más claramente explican, en términos estadísticos, el surgimiento de la violencia (Klevens, 2001). Sin embargo, ino es el hecho de imponer prohibiciones a la pareja una forma concreta de ejercer violencia contra ella? Si es así, ¿no es entonces del todo esperable que ambas "variables" estén fuertemente asociadas? El problema de fondo es que, con toda probabilidad, no estamos aquí frente a "dos" variables, sino más bien frente a dos manifestaciones de la misma variable.

En otro caso, se reporta como variables independientes el tener actitudes que justifiquen el uso de la violencia y el contar con escasas habilidades para resolver los conflictos interpersonales (Moreno, 1999). Pero ¿no es el uso de la violencia una demostración de una falta de habilidades para resolver un conflicto en otros términos? Si es así, ¿qué aporta realmente, en términos de esclarecimiento de las causas del problema, el sugerir que la dificultad de no ser violento es lo que explica la conducta violenta?
Y, en un caso más, se señala que existe una fuerte asociación entre la adopción de normas que justifican el uso de la violencia y el uso de la violencia propiamente tal, si bien se reconoce que, en este estudio, "las normas y actitudes son medidas por la capacidad de la pareja de controlarse a sí misma y de actuar de una manera no violenta" (Briceño-Leon et al., 1999:132). Esto es, el autor mismo reconoce que una misma medición le sirve para construir dos variables, a saber, la conducta violenta y, en su defecto, la existencia de normas y actitudes contrarias a la violencia. Estrategia que contradice el principio metodológico que postula la necesaria independencia de las mediciones que se busca asociar, si se quiere contar con ciertas garantías, sobre la validez de la asociación encontrada.

Es verdad que resulta problemático diferenciar dónde termina la desigualdad de género y dónde comienza la violencia de género, pues esta última no es sino la consecuencia extrema de la primera (A. Guezmes, comunicación personal). Pero éste, siendo en principio un problema teórico y conceptual, no nos autoriza a incurrir en confusiones operacionales tan evitables como las señaladas.

Obviedad de los hallazgos y las recomendaciones

Junto al problema anterior, una segunda cuestión que preocupa se refiere a la frecuente obviedad tanto de los hallazgos como de las recomendaciones que se derivan de los mismos. Sorprende, por ejemplo, que en una investigación se reporte que entre estudiantes de nivel preparatoria los principales factores de riesgo para incurrir en conductas antisociales o violentas son el hecho de ser hombre, consumir alcohol y utilizar otro tipo de drogas (Juárez et al., 1998). ¿No lo sabíamos?

En otros trabajos, tras la presentación de los hallazgos, se formula una serie de recomendaciones aparentemente orientadas a combatir y prevenir la violencia en la pareja. Como los estudios muestran que el desbalance de poder en la pareja se asocia a este problema, se recomienda... ¡balancear dicho poder! Y en el mismo tenor, es posible encontrar recomendaciones para cambiar las actitudes, reducir los factores que estresan a la pareja y enseñar a los hombres a manejar conflictos sin violencia (Firestone et al., 2000; Moreno, 1999).

Sin duda les asiste alguna razón a todas estas sugerencias de intervención. Pero no es posible evitar la sensación de que es poco lo que contribuyen a esclarecer la naturaleza del pro- 
blema. Pues la pregunta de investigación que seguimos sin resolver es precisamente qué sostiene el desbalance de poder entre hombres y mujeres, qué alimenta las actitudes agresivas, por qué el estrés se asocia a la violencia, etc. Y como lo hemos venido haciendo a lo largo de todo este trabajo, sostenemos que es la falta de una teoría social más sólida que sirva de anclaje a toda esta investigación lo que confiere esa sensación de orfandad a las recomendaciones que se formulan.

\section{Vacío de investigación con hombres}

Finalmente, junto a la dificultad para distinguir con claridad las variables dependientes de las independientes, junto a la producción de resultados inconsistentes que contribuyen a incrementar nuestro desconcierto, y al lado de los hallazgos reiterativos y obvios, la investigación sobre el problema registra un asombroso vacío: la investigación "río arriba”, centrada en el origen del problema, la que tome a los propios hombres como los sujetos de la investigación y explore en ellos las preguntas que nos inquietan.

Sólo recientemente se han iniciado los estudios sobre "masculinidad" en América Latina, pero falta aún mucho por explorar en este sentido. No es difícil entender por qué las investigaciones se han centrado hasta ahora en las mujeres. Primero, porque son el grupo agredido y es urgente trabajar en la defensa y vigencia de sus derechos. Segundo, porque son un grupo mucho más fácilmente entrevistable, que el de los hombres. Pero, claramente, el problema no empieza con ellas. Sólo en la medida en que la investigación sobre la violencia se amplíe hacia los hombres y hacia la interacción entre hombres y mujeres, estaremos en condiciones de dar con nuevos hallazgos relevantes.

\section{Conclusión}

Para concluir, retomemos justo lo que señalábamos en el párrafo anterior. ¿Por qué es difícil hacer investigación sobre violencia con hombres? En primer lugar, por supuesto, porque se quiere evitar generar situaciones de riesgo que pondrían en peligro, una vez más, la seguridad de las mujeres. Pero, en segundo lugar, y esto no es menos importante, porque los hombres se resisten más a hablar del tema que las mujeres. En el reporte de resultados del proyecto ACTIVA (Fournier et al., 1999) se señala que más hombres que mujeres declinaron participar. La imposibilidad de la investigación está asociada al poder, es decir, al mismo mecanismo que genera el problema (la violencia) que se desea explicar. Esa imposibilidad, a su vez, puede estar contribuyendo a perpetuar el carácter simultáneamente pobre y reiterativo de la investigación actual, pues a fuerza de no poder medir lo que se quiere investigar se termina por investigar lo que se puede medir. "Corremos el peligro, por tanto, de recurrir, para concebir a la dominación masculina, a unos modos de pensamiento que ya son el producto de la dominación. Sólo podemos confiar en salir de ese círculo si encontramos una estrategia práctica para efectuar una objetivación del tema de la objetivación científica" (Bourdieu, 2000b:17). El gran reto de la investigación actual sobre el problema de la violencia contra las mujeres radica, a nuestro juicio, en la solución de este problema. Dicha solución pasa por dos cuestiones: (a) el desarrollo de investigaciones que recuperen el carácter relacional de la violencia en contextos intermedios, como la familia, la pareja y la calle (Gomes, 1994); y (b) recordar que las ciencias sociales no son sólo un instrumento para hacer nuestra investigación: son el núcleo de nuestro quehacer, y constituyen la única posibilidad de salir del enorme atolladero cognoscitivo en que nos encontramos. La pobreza y las limitaciones de los resultados de investigación hasta ahora alcanzados en el tema de la violencia contra las mujeres son consecuencia del abandono de nuestro propio patrimonio: a menos que nos comprometamos de lleno con el corpus conceptual y metodológico de nuestras propias disciplinas, seguiremos oscilando entre la trivialidad y la perplejidad. 


\section{Agradecimientos}

Los autores desean agradecer al Dr. Carlos Cáceres, cuya invitación para el VI Congreso Latinoamericano de Ciencias Sociales y Salud fue el punto de arranque para la elaboración de este trabajo. Agradecemos también la entusiasta colaboración de Rodrigo Madrigal Briones, quien trabajó en la búsqueda de material bibliográfico y en su sistematización.

\section{Referências}

ALVARO-ZALDIVAR, G.; SALVADOR-MOYSEN, J.; ESTRADA-MARTINEZ, S. \& TERRONES-GONZALEZ, A., 1998. Prevalencia de violencia doméstica en la ciudad de Durango. Salud Pública de México, 40: 481-486.

ALVES-CABRAL, M. A., 1999. Prevenção da violência conjugal contra a mulher. Ciência \& Saúde Coletiva, 41:183-191.

ANONYMOUS, 1984-1985. Mujer y violencia. Fempress, 8.

ANONYMOUS, 1987. Violencia en casa. Fempress, 11. ANONYMOUS, 1992. Mujer. Fempress, 127.

ARCOS, E.; MOLINA, I.; REPOSSI, A.; UARAC, M.; RITTER, P. \& ARIAS, L., 2000. Detección de Violencia Doméstica en Mujeres Embarazadas. 20 Junio 2002 <http://www.hsph.harvard.edu/grhf/_Spanish/ gender/docs/deteccion.html>.

BARROSO, I., 1988. Hostigamiento sexual. Fem, 12:916.

BEDOLLA, P., 1987. Hostigamiento sexual en los espacio laborales. Casa del Tiempo, 8:13-17.

BEDREGAL, X., 1991a. El Centro de Apoyo a la Mujer (CAM) de Colima. In: Hilos, Nudos y Colores en la Lucha Contra la Violencia Hacia las Mujeres (X. Bedregal, I. Saucedo, F. Riquer, org.), pp. 143-168, México, DF: Centro de Investigación y Capacitación de la Mujer.

BEDREGAL, X., 1991b. Anexo Jurídico. In: Hilos, $\mathrm{Nu}$ dos y Colores en la Lucha Contra la Violencia Hacia las Mujeres (X. Bedregal, I. Saucedo, F. Riquer, org.), pp. 207-248, México, DF: Centro de Investigación y Capacitación de la Mujer.

BEDREGAL, X.; SAUCEDO, I. \& RIQUER, F., 1991. Algunos hilos, nudos y colores en la lucha contra la violencia hacia las mujeres. In: Hilos, Nudos y Colores en la Lucha Contra la Violencia Hacia las Mujeres (X. Bedregal, I. Saucedo \& F. Riquer, org.), pp. 39-84, México, DF: Centro de Investigación y Capacitación de la Mujer.

BOUDIEU, P., 2000b. La Dominación Masculina. Barcelona: Editorial Anagrama.

BOURDIEU, P., 2000a. Poder, Derecho y Clases Sociales. Bilbao: Desclée.

BRICEÑO-LEON, R.; CAMARDIEL, A.; AVILA, O. B. \& DeARMAS, E., 1999. Domestic violence in Caracas: Social and cultural predictors. Acta Científica Venezolana, 50:132-143.
BURIN, M. \& MELER, I., 1998. Género y Familia. Poder, Amor y Sexualidad en la Construcción de la Subjetividad. Buenos Aires: Paidós.

BUVINIC, M.; MORRISON, A. R. \& SHIFTER, M., 1995. La violencia en las Américas: Marco de acción. In: El Costo del Silencio. Violencia Doméstica en las Américas (A. R. Morrison \& M. L. Biehl, org.), pp. 334, New York: Banco Interamericano de Desarrollo.

CASTRO, R. \& BRONFMAN, M., 1993. Teoría feminista y sociología médica: Bases para una discusión. Cadernos de Saúde Pública, 9:375-394.

CASTRO, R.; CAMPERO, L.; HERNANDEZ, B. \& LANGER, A., 2000. A study on maternal mortality in Mexico through a qualitative approach. Journal of Women's Healh \& Gender-Based Medicine, 9:679-690.

CASTRO, R. \& ERVITI, J., 2002. La violación de los derechos reproductivos durante la atención del parto: Un estudio introductorio. En: Género y Políticas de Salud en México. Una Propuesta para el Plan Nacional de Salud (G. Espinoza, M. P. López, B. Rico \& P. Mercado, org.), México, DF: Organización Panamericana de la Salud/Fondo de Desarrollo de Naciones Unidas para la Mujer.

CASTRO, R.; PEEK-ASA, C.; GARCIA, L. \& RUIZ, A., 2001. Social factors associated with violence against pregnant women: A comparative study between Morelos (Mexico) and California (United States). American Journal of Epidemiology, 153 (Sup.):243.

CASTRO, R. \& RUIZ, A., 2002. Violencia contra mujeres embarazadas en el estado de Morelos: Hallazgos preliminares. In: Género y Políticas de Salud en México. Una Propuesta para el Plan Nacional de Salud (G. Espinoza, M. P. López, B. Rico \& P. Mercado, org.), México, DF: Organización Panamericana de la Salud/Fondo de Desarrollo de Naciones Unidas para la Mujer.

CLADEM (Comite de América Latina y el Caribe para la Defensa de los Derechos de la Mujer), 1998. Silencio y Complicidad. Violencia Contra las $\mathrm{Mu}$ jeres en los Servicios Públicos de Salud en el Perú. Lima: CLADEM/Centro Legal para Derechos Reproductivos y Políticas Públicas.

CORSI, J., 1994. Violencia Familiar. Una Mirada Interdisciplinaria Sobre un Grave Problema Social. Buenos Aires: Paidós. 
DESLANDES, S. F., 2000. Caracterização dos casos de violência doméstica contra a mulher atendidos em dois hospitais públicos do Rio de Janeiro. Cadernos de Saúde Pública, 16:129-137.

DIAZ-OLAVARRIETA, C.; CAMPBELL, J.; GARCIA-DELA-CADENA, C.; PAZ, F. \& VILLA, A. R., 1999. Domestic violence against patients with chronic neurologic disorders. Archives of Neurology, 56:681685.

DIAZ-OLAVARRIETA, C.; PAZ, F; DE LA CADENA, C. G \& CAMPBELL, J., 2001. Prevalence of intimate partner abuse among nurses and nurses' aides in Mexico. Archives of Medical Research, 32:79-87.

DINIZ, S. G. \& D'OLIVEIRA, A. F., 1998. Gender violence and reproductive health. International Journal of Gynaecology and Obstetrics, 63(Sup. 1):3342.

DUARTE, P., 1992. La violación y su impacto en la salud integral de la mujer. Aspectos psicológicos. In: III Congreso Nacional de Investigación en Salud Pública, Anales, p. 154, Cuernavaca: Instituto Nacional de Salud Pública.

ELLSBERG, M.; CALDERA, T.; HERRERA, A.; WINKVIST, A. \& KULLGREN, G., 1999. Domestic violence and emotional distress among Nicaraguan women: Results from a population-based study. American Psychologist, 54:30-36.

ELLSBERG, M.; PEÑA, R.; HERRERA, A.; LILJESTRAND, J. \& WINKVIST, A., 2000. Candies in hell: Women's experiences of violence in Nicaragua. Social Science and Medicine, 51:1595-1610.

FASSLER, C., 1999. Violencia doméstica. Algunas reflexiones. In: Frente al Silencio. Testimonios de la Violencia en Latinoamérica (G. Araujo, O. Desatnik \& L. Fernández, org.), pp. 253-264, México, DF: Universidad Autónoma Metropolitana-Xochimilco.

FOURNIER, M.; RIOS, R.; ORPINAS, P. \& PIQUETCARNEIRO, L., 1999. Multicenter study on Cultural Attitudes and Norms towards violence (ACTIVA project): Methodology. Revista Panamericana de Salud Pública, 5:222-231.

GAFFNEY, K. F.; CHOI, E.; YI, K.; JONES, G. B.; BOWMAN, C. \& TAVANGAR, N. N., 1997. Stressful events among pregnant Salvadoran women: A cross-cultural comparison. Journal of Obstetric, Gynecologic, and Neonatal Nursing, 26:303-310.

GARZA-AGUILAR, J. \& DIAZ-MICHELE, E., 1997. Elementos para el estudio de la violación. Salud Pública de México, 39:539-545.

GIDEENS, A., 1995. La Constitución de la Sociedad. Bases para la Teoría de la Estructuración. Buenos Aires: Amorrortu Editores.

GIFFIN, K., 1994. Violência de gênero, sexualidade e saúde. Cadernos de Saúde Pública, 10:146-155.

GOMES, R., 1994. A violência enquanto agravo à saúde de meninas que vivem nas ruas. Cadernos de Saúde Pública, 10:156-167.

GOODWIN, M. M.; GAZMARARIAN, J. A.; JOHNSON, C. H.; GILBERT, B. C.; SALTZMAN, L. E. \& THE PRAMS WORKING GROUP, 2000. Pregnancy intendedness and physical abuse around the time of pregnancy: Findings from the pregnancy risk assessment monitoring system, 1996-1997. Maternal and Child Health Journal, 4:85-92.

GRANADOS, S. M., 1996. Salud Reproductiva y Violencia Contra la Mujer: Un Análisis Desde la Per- spectiva de Género; El Caso de la Zona Metropolitana de Monterrey. México, DF: Asociación Mexicana de Población.

HEISE, L., 1994. Violencia Contra la Mujer. La Cara Oculta de la Salud. Washington, DC: Programa Mujer, Salud y Desarrollo, Organización Panamericana de la Salud.

HERRERA, C. \& CAMPERO, L., 2000. Violencia, mujeres y VIH/SIDA. Grupo Latinoamericano de Trabajo Sobre Mujeres y SIDA - Boletín Informativo, 4:3-4.

JUAREZ, S.; MEDINA-MORA, E.; BERENZON, S.; VILLATORO, J. A.; CARRENO, S.; LOPEZ, E. K.; GALVAN, J. \& ROJAS, E., 1998. Antisocial behavior: Its relation to selected sociodemographic variables and alcohol and drug use among Mexican students. Substance Use \& Misuse, 33:1437-1459.

KLEVENS, J., 2001. Violencia física contra la mujer en Santa Fe de Bogotá: Prevalencia y factores asociados. Revista Panamericana de Salud Pública, 9:78-83.

LARRAIN, S., 1993. Estudio de Frecuencia de la Violencia Intrafamiliar y la Condición de la Mujer Chilena. Santiago de Chile: Organización Panamericana de la Salud.

LARRAIN, S. \& RODRIGUEZ, T., 1993. Los orígenes y el control de la violencia doméstica en contra de la mujer. In: Género, Mujer y Salud en las Américas (E. Gómez, org.), pp. 48-60, Washington, DC: Organización Panamericana de la Salud.

MATTSON, S. \& RODRIGUEZ, E., 1999. Battering in pregnant Latinas. Issues in Mental Health Nursing, 20:405-422.

MENEGHEL, S. N.; CAMARGO, M.; FASOLO, L. R.; MATTIELLO, D. A.; SILVA, R. C.; SANTOS, T. C.; DAGORD, A. L.; RECK, A.; ZANETTE, L.; SCOTTILI, M. \& TEIXEIRA, M. A., 2000. Mulheres cuidando de mulheres: Um estudo sobre a Casa de Apoio Viva Maria, Porto Alegre, Rio Grande do Sul, Brasil. Cadernos de Saúde Pública, 16:747-757.

MILLER, R. S., 1978. The social construction and reconstruction of physiological events: Acquiring the pregnancy identity. Studies in Symbolic Interaction, 1:181-204.

MOGROVEJO, N., 1989. La violación en el Perú. Realidad y tratamiento jurídico. In: $i Y$ Hasta Cuando Esperaremos Manda-Dirun.Dirun-Dán?. Mujer y Poder en América Latina (A. Koschutzke, org.), pp. 241-251, Caracas: Editorial Nueva Sociedad.

MOORE, M., 1999. Reproductive health and intimate partner violence. Family Planning Perspectives, 31:302-312.

MORENO, F., 1999. Violence between couples. Revista Panamericana de Salud Pública, 5:245-258.

OLIVEIRA, O., 1998. Familia y relaciones de género en México. In: Familias y Relaciones de Género en Transformación (B. Schmukler, org.), pp. 23-52, México, DF: Population Council/EDAMEX.

PEREZ, L. R., 2000. Fiesta as tradition, fiesta as change: Ritual, alcohol, and violence in a Mexican community. Addiction, 95:365-73.

PROFAMILIA, 1990. La Violencia y Los Derechos Humanos de La Mujer. Encuesta de Prevalencia Demografía y Salud. Violencia Intrafamiliar. Bogotá: Servicio de Consultoría Jurídica.

RAMIREZ, J. C. \& URIBE, G., 1993. Mujer y violencia: 
Un hecho cotidiano. Salud Pública de México, 35:148-160.

RAMIREZ, F., 2000. Violencia Masculina en El Hogar. México, DF: Editorial Pax.

RAMIREZ, J. C. \& PATIÑO, M. C., 1996. Mujeres de Guadalajara y violencia: Resultados de un estudio piloto. Cadernos de Saúde Pública, 12:405-409.

RIQUER, F., 1991. La agresión masculina contra la mujer: Notas para seguir pensando. In: Hilos, $\mathrm{Nu}$ dos y Colores en la Lucha Contra la Violencia Hacia las Mujeres (X. Bedregal, I. Saucedo \& F. Riquer, org.), pp. 17-38, México, DF: Centro de Investigación y Capacitación de la Mujer.

RIQUER, F., 1992. Violencia masculina hacia el género femenino: Un intento de conceptualización. In: III Congreso Nacional de Investigación en Salud Pública, Anales, p. 153, Cuernavaca: Instituto Nacional de Salud Pública.

RIQUER, F., 1995a. Violencia y Salud de la Mujer. Oportunidades y Obstáculos para su Atención. El caso de México. Resultados preliminares. México, DF: Grupo de Trabajo sobre Salud y Violencia Sexual y Doméstica, Programa Salud Reproductiva y Sociedad, Colegio de México.

RIQUER, F., 1995b. Género y violencia intrafamiliar. In: XXXIV Congreso Nacional de Psicoanálisis, Memorias, p. 103, Morelia: Sociedad Mexicana de Psicoanálisis.

RIQUER, F.; SAUCEDO, I. \& BEDOLLA, P., 1996. Agresión y violencia contra el género femenino: Un asunto de salud pública. In: Mujer: Sexualidad y Salud Reproductiva en México (A. Langer \& K. Tolbert, org.) pp. 247-287, México, DF: Population Council/EDAMEX.

ROJAS, R., 1991. Experiencia en San Cristóbal de las Casas, Chiapas. In: Hilos, Nudos y Colores en la Lucha Contra la Violencia Hacia las Mujeres (X. Bedregal, I. Saucedo \& F. Riquer, org), pp. 169178, México, DF: Centro de Investigación y Capacitación de la Mujer.

RUBIN, G., 1975. The traffic in women: Notes on the "political economy" of sex. In: Toward an Anthropology of Women (R. R. Reiter, ed.), pp. 157-210, New York: Monthly Review Press.
SAUCEDO, I., 1991. El difícil camino a la individuación: Procesos grupales en el feminismo. In: Hilos, Nudos y Colores en la Lucha Contra la Violencia Hacia las Mujeres (X. Bedregal, I. Saucedo \& F. Riquer, org.), pp. 85-142, México, DF: Centro de Investigación y Capacitación de la Mujer.

SAUCEDO, I., 1996. Mujer y violencia. Entorno familiar y social. In: Humanismo, Mujer, Familia y Sociedad (M. García \& A. Pulido, org.), pp. 36-56, México, DF: Sociedad Provalores Humanos/Instituto Nacional de la Nutrición Salvador Zubirán/ Programa Interdisciplinario de Estudios de la Mujer.

SILVA, U., 1986. Eramos asombrosamente felices. Cuadernos de la Mujer, 3:3-13.

SZASZ, I., 1999. Género y salud. Propuestas para el análisis de una relación compleja. In: Salud, Cambio Social y Política. Perspectivas desde América Latina (M. Bronfman \& R. Castro, org.), pp.109122, México, DF: EDAMEX.

THE BRITISH COUNCIL, 1999. La Violencia Contra las Mujeres: Un Documento Informativo sobre Cuestiones y Respuestas Internacionales. Manchester: The British Council.

TOLBERT, K. \& ROMERO, M., 1996. Violencia sexual y familiar en México. Resultados de una encuesta en San Miguel de Allende. Hombres y Familias, 3:19-24.

TURNER, B. S., 1987. Medical Power and Social Knowledge. London: Sage Publications.

VALDEZ, R. \& SHRADER, E., 1992. Características y análisis de la violencia doméstica en México: El caso de una microregión de Cd. Nezahualcóyot. In: Aún la Luna a Veces Tiene Miedo (Centro de Investigación y Lucha contra la Violencia Doméstica, org.), pp. 35-49, México, DF: Centro de Investigación y Lucha contra la Violencia Doméstica.

WATTS, C. H. \& SHRADER, E., 1998. How to do (or not to do)... The genogram: A new research tool to document patterns of decision-making, conflict, and vulnerability within households. Health Policy and Planning, 13:459-464.

Recibido el 22 de noviembre de 2001

Versión final presentada el 6 marzo de 2002

Aprobado el 12 de julio de 2002 